\title{
Need, Demand and Supply of Health Care in Regards to Growing Emerging and Reemerging Diseases in Sub-Saharan Africa
}

\author{
Article by Medang Raphael \\ Ph.D. in Public Health, Texila American University, Cameroon \\ E-mail: rmedang@metabiota.com
}

\section{Introduction}

Need in relation to health care, has generally been defined as the amount of medical care that medical experts believe a person should have to remain or become as healthy as possible, based on a current medical knowledge. This is also true for the individual and for the entire community. Thus, if a problem, a barrier or difficulty continues to exist on the path to accession to a higher level of health, the need exists. In "The definition and identification of need for health care ${ }^{1}$," Acheson stated that there exist four phenomena underlying health needs including: - the risk of pain and illness (in the sense of discomfort, or "malaise") - the risk of sickness - the inadequacy and deficiency - the mortality risk. Finally, although certain health needs are expressed, they do not correspond to real needs. This would provide different areas related to health interventions, including:

- The ideal zone (a need corresponds to an expressed demand and adequate health action);

- The area of non-use (a health action not corresponding to any demand);

- Non-use of family planning services in both high population growth areas but also high poverty

- The waste area (a health action corresponding to a demand but with no corresponding need);

- The prescription of laboratory tests and other unnecessary medical procedures in response to public solicitation, especially persons holding health insurance

- The discontent area (a demand triggered by a real need, but to which no health action is taken);

- The recurrence of malaria cases in one region, and the desire by local residents, to have currently non-existent measures, to be taken by health and government authorities, on the environmental and sanitation plan.

The following figure describes the triad related to health care needs, demands and responses

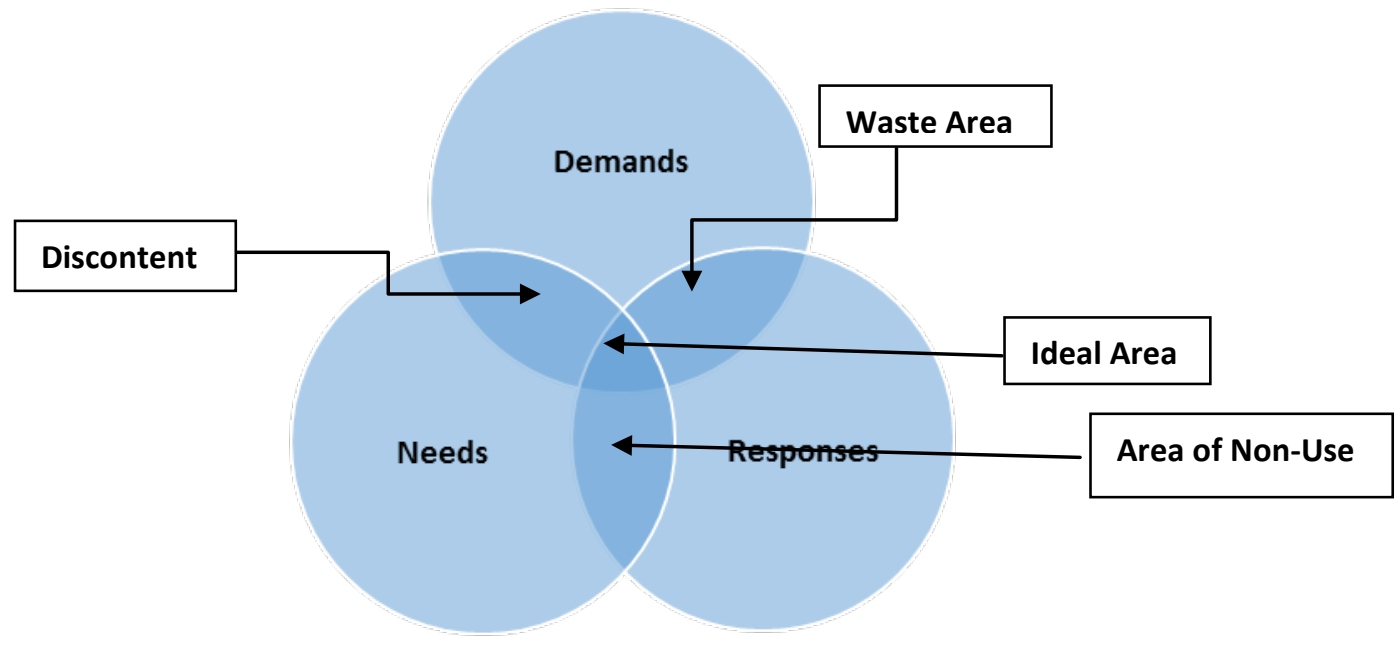

Graph 1. The Triad of Public Health

\footnotetext{
${ }^{1}$ Acheson, 1978
} 
As we can see, needs, demands, and responses (that can also be identified as health supply) are then interconnected. This means that there is a real need for health systems to be able to adapt and to react positively to new, emerging and reemerging situations in relation to the health of populations. This is especially true in low-income countries such as those in Sub-Saharan Africa.

These emerging infections (Els) are most of the time defined as "infections that have newly appeared in a population or have existed previously but is rapidly increasing in incidence or geographical range.” This means that the most important point here is the increase in incidence, that is to say the new cases of new infections within a population. These infections are usually caused by newly identified agents, such as HIV or the Ebola Virus. Factors that contribute to the emergence of these diseases are numerous and can be listed as follow: - inadequate control in water supply, international travel; increased use of child-care facilities - interruption of immunization programs due to political changes - drug resistance; favorable conditions for mosquito vector - refusal to vaccinate based on fears that the vaccine is not safe; other possible factors; decreased vaccine efficacy or waning immunity among vaccinated adults - breakdown in public health measures; changes in land use; travel; - dam construction - ecological changes - insecticide resistance - poverty and social inequality - war and famine - etc. This increase in disease incidence, will obviously lead to the increase in demand for health services.

In a context of scarcity of financial resources, populations will face an increase in terms of varieties and number of infections they will face, but also a health system in which they are the main donors. As a matter of fact, talking of health financing in Sub-Saharan Countries, there's a common concept which states that health insurance helps reduce out-of-pocket costs. Unfortunately, out-of-pocket continues to be the main pillar of the health system funding sources in some countries, such as Cameroon, a central African country. In Cameroon Economic Update, a twice-yearly World Bank publication designed to promote dialogue on various aspects of the country's outlook, the sixth issue was devoted to the subject of health. According to the publication, even though Cameroon's proportion of doctors (1.9 per 1,000 inhabitants) is twice the minimum recommended by the World Health Organization, the country's health statistics are paradoxically behind the curve. Life expectancy for Cameroonians has decreased by about two years since 1990, while it has increased by an average of five years in the rest of sub-Saharan Africa. Worldwide, Cameroon is also among the countries where the mortality rate for children under five years of age (122 deaths per 1,000 live births) has decreased the least. To this end and according to a report issued by the World Bank in 2013, Cameroon (a country with over 20 million inhabitants), "spends more money on health than any other sub-Saharan country (except South Africa): US\$61 per capita, as opposed to US\$51 on average. It is Cameroonians themselves, however, who shoulder the majority of this financial burden. "Out of those \$61, the State finances only $\$ 17$; and out of that sum, \$8 comes from international donors," notes Raju Jan Singh, the World Bank lead economist for Central Africa and the main author of the report. "This means there is a strong correlation between health statistics and revenue statistics," he adds, "with well-off households and wealthy regions having better access to health services." This means obviously that, there exist problems related not only to affordability and access to health care, but also in a more general way, those of equity. The health system is heavily based on the out of pocked financing system by individuals and households. And as such, only those who are able to pay will be able to obtain health care, particularly health care of good quality. However, mechanisms such as those of health or social insurance coverage are very uncommon here. Only a few people exercising in the formal sector are eligible for health insurance mechanisms or some other social insurance. According to the results of the Second Survey of Employment and the Informal Sector in Cameroon (EESI 2) 2010, "the main problem of the labor market in Cameroon is not unemployment but underemployment. Nearly three out of four workers are underemployed, underemployment, which is mainly due to low pay, since $70.3 \%$ of workers earn less than the legal monthly minimum wage which is 28,500 CFA francs. That's as many people that are practically excluded from the formal and 
modern system of health care. Their only recourse then remains in the so-called traditional medicine or self-medication.

The issues related to access to services are not only financial, but also geographic. In this regard, the report of the World Bank emphasizes in particular that "The geographic disparities are striking: $40 \%$ of the country's doctors practice in the Centre region (which includes Yaoundé, the capital), where only $18 \%$ of the population lives. On the other hand, the Far North region, which also holds $18 \%$ of the population, employs only $8 \%$ of Cameroon's doctors. It can be seen that the main consequence of the health system as currently implemented and as it currently operates in Cameroon, is that of exclusion. "Some form of exclusion appears in respect to the poorest social strata, especially the rural population, those exercising in the informal sector in both urban and rural areas, and people under the general underemployment sectors.

Two major challenges thus emerge here, not only in relation to the population, but also in relation to the public health system. The first challenge is related to the ability of people to seek services, seek and obtain health services as regard to their health needs in a context of emerging infections. The second challenge is related to the capacity of the health system to adequately respond to the needs of its population, still in the context of emerging and reemerging diseases, knowing also that we are in a context where the most economically disadvantaged are the most vulnerable to disease and other illnesses.

Based on all the above, our focus in terms of research questions were the following; "What are the current trends concerning health care, public surveillance and research activities related to emerging and reemerging diseases?” - What are the procedures, the practices and state of situation as regards to needs and demand of health care concerning emerging and reemerging diseases? - What are some research activities, as well as public surveillance activities currently undertaken in regards to emerging and reemerging diseases? By answering these questions, we might be able not only to put together a number of data, knowledge and practices related to emerging and reemerging diseases, but also the way in which these scourges are taken care of and brought under control by health systems around the world.

The general objective was to determine from the literature, the state of the art concerning needs and demand of health care, public health surveillance and fundamental research activities related to emerging and reemerging diseases. On this basis, the specific objectives were to: -Describe overall knowledge on emerging and reemerging diseases. - Describe state of needs and demand of health care as regards to emerging and reemerging diseases - Describe some of the procedures involved in scientific research as well as public health surveillance of emerging and reemerging diseases.

\section{Methodology}

We carried out a historical literature review of published and grey literature on various aspects of emerging and reemerging diseases, including needs, demands and responses. It was indeed a literature review from 1995 to 2015, that is to say publication spanning over 20 years. Thirty-seven scientific and published articles, key documents and reviews were consulted through PubMed, Medline and Google Scholar, to screen relevant information on emerging and reemerging infections, as well as needs, demands and provision of services related to this. Out of these, a total number of 21 were eligible for this review. The chosen articles and publications were selected and identified using terms such as emerging diseases in Africa, reemerging diseases in Africa, demand for health care, and provision of health care. Only articles, reports, key documents and reviews published in English from 1995 to 2015 were selected (except the key document related to the definition and identification of need for health care). However, we did not assess the methods or reliability of the various estimates or data presented here, but tried to collect as much information as possible in order to have a comprehensive review of the situation with regards to emerging and reemerging diseases, needs and demands for services, as well as provision of health care. 
Texila International Journal of Public Health

Volume 4, Issue 4, Dec 2016

\section{Results}

As some authors have already reported, several factors may be at the origin of emerging and reemerging diseases in the World, and more particularly in Sub-Saharan Africa. In a publication entitled "Factors in the Emergence of Infectious Diseases"," "Emerging" infectious diseases were defined as infections that have newly appeared in a population or have existed but are rapidly increasing in incidence or geographic range. Ecological changes, including those due to agricultural (see Table 1) or economic development, are among the most frequently identified factors in emergence. They are especially frequent as factors in outbreaks of previously unrecognized diseases with high case-fatality rates, which often turn out to be zoonotic introductions. Ecological factors usually precipitate emergence by placing people in contact with a natural reservoir or host for an infection hitherto unfamiliar but usually already present (often a zoonotic or arthropod-borne infection), either by increasing proximity or, often, also by changing conditions so as to favor an increased population of the microbe or its natural host.

\footnotetext{
${ }^{2}$ Morse, 1995
} 


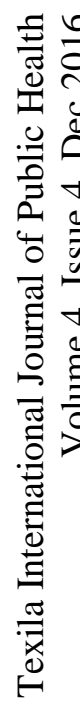

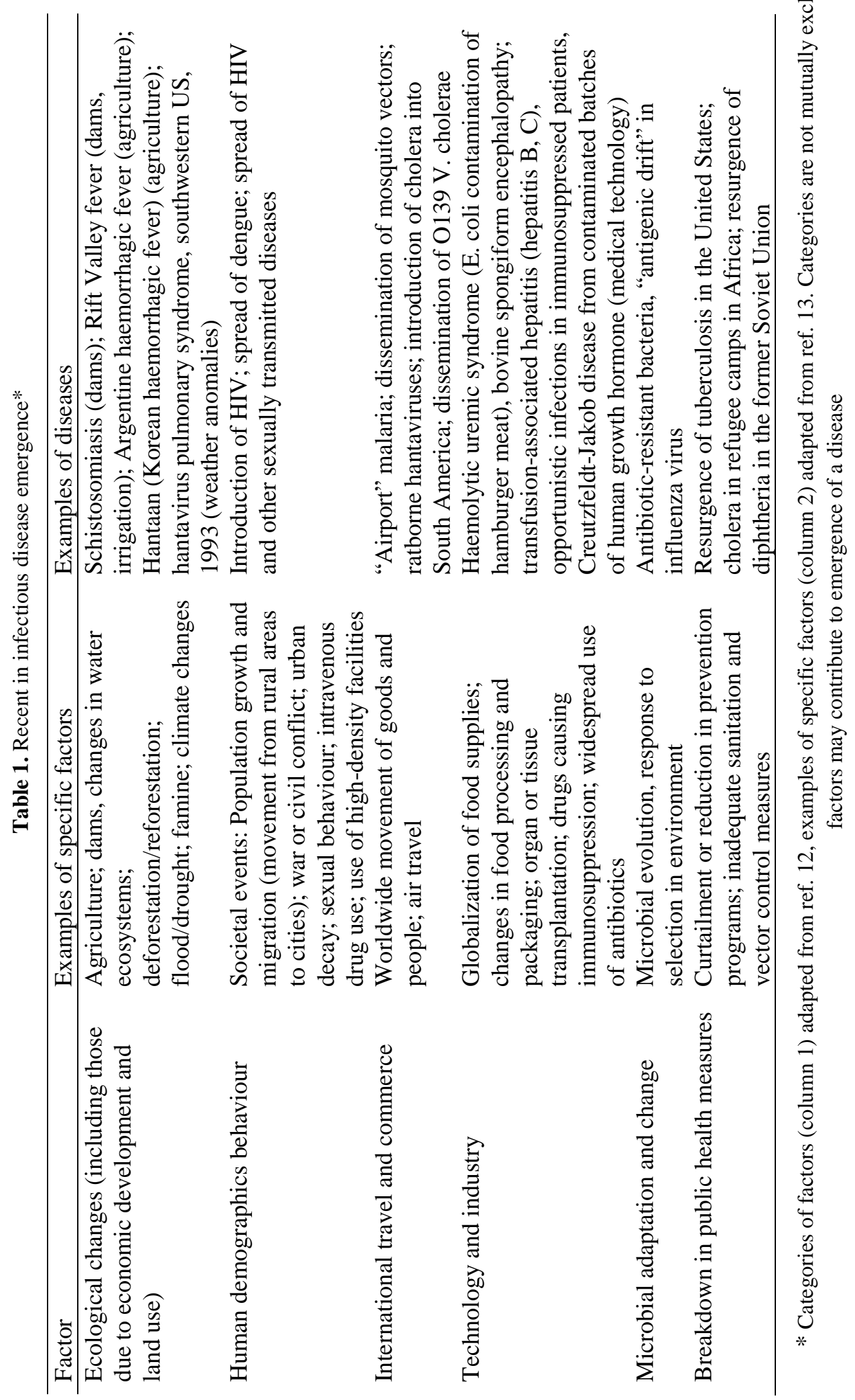


As demonstrated by influenza epidemics, under suitable circumstances, a new infection first appearing anywhere in the world could traverse entire continents within days or weeks. Recent examples of emerging diseases in various parts of the world include HIV/AIDS; classic cholera in South America and Africa; cholera due to Vibrio cholerae 0139; Rift Valley fever; hantavirus pulmonary syndrome; Lyme disease; and haemolytic uremic syndrome, a foodborne infection caused by certain strains of Escherichia coli (in the United States, serotype O157:H7). Although these occurrences may appear inexplicable, rarely if ever do emerging infections appear without reason. Some of the specific emerging and re-emerging diseases were identified and classified as follow:

- Viral: Argentine, Bolivian haemorrhagic fever - Bovine spongiform encephalopathy (cattle) Changes in agriculture favouring rodent host - Dengue haemorrhagic fever - Ebola - Hepatitis B, C - HIV - HTLV - Influenza (pandemic) - Lassa fever - Rift Valley fever - Yellow fever

- Bacterial: Brazilian purpuric fever (Haemophilus influenzae, biotype aegyptius) - Cholera Helicobacter pylori - Haemolytic uremic syndrome (E. coli O157:H7) - Legionella (Legionnaires' disease) - Lyme borreliosis (Borrelia burgdorferi) - Streptococcus, group A Toxic shock syndrome (Staphylococcus aureus)

- Parasitic: Cryptosporidium, other waterborne pathogens - Malaria (in "new" areas) Schistosomiasis and other helminthiasis.

In terms of prevalence, geographic distribution and disease burden, as well as types of diseases, the diseases labelled as neglected account among the most emerging ones. In fact, according to the result of a study entitled "Neglected Tropical Diseases in Sub-Saharan Africa: Review of Their Prevalence, Distribution, and Disease Burden ${ }^{3}$, many neglected tropical diseases (NTDs) also appear to be emerging diseases. According to the authors, "the neglected tropical diseases (NTDs) are the most common conditions affecting the poorest 500 million people living in sub-Saharan Africa (SSA), and together produce a burden of disease that may be equivalent to up to one-half of SSA's malaria disease burden and more than double that caused by tuberculosis.” Today, the world's greatest concentration of poverty occurs in sub-Saharan Africa (SSA). According to a recent World Bank analysis, $51 \%$ of the population of SSA lives on less than US $\$ 1.25$ per day, and $73 \%$ of the population lives on less than US\$2 per day (see Table 2).

Table 2. Poverty in Sub-Saharan Africa.

\begin{tabular}{lc}
\hline Percentage of SSA population living on less than US\$1.25 per day & $51 \%$ \\
Total SSA population living on less than \$1.25 per day & 390.6 million \\
Percentage of world's population living on less than US\$1.25 per day in SSA $28 \%$ \\
Percentage of SSA population living on less than US\$2 per day & $73 \%$ \\
Total SSA population living on less than \$2 per day & 556.7 million \\
Percentage of world's population living on less than US\$2 per day in SSA & $22 \%$ \\
\hline
\end{tabular}

From http://www-wds.worldbank.org.

Still according to the above-mentioned article, specific information on the prevalence, distribution, and disease burden resulting from the NTDs in SSA are as follow:

- Helminth infections, especially the soil-transmitted helminth (STH) infections, schistosomiasis, and the filarial infections and onchocerciasis, are the most common NTDs in SSA, followed by trachoma and other bacterial infections.

- Human African trypanosomiasis (HAT) and leishmaniasis are the most common serious protozoan infections, especially in areas of conflict where these diseases emerge in the setting of inadequate housing and forced migrations.

\footnotetext{
${ }^{3}$ Hotez \& Kamath, 2009
} 
- However, there are more than a dozen important NTDs, including the protozoan infections, amoebiasis and toxoplasmosis; bacterial infections such as Salmonella infections (both typhoid fever and non-typhoidal salmonellosis), the tick-borne zoonoses, and yaws; and viral infections such as Rift Valley fever, for which there is insufficient information available in order to estimate their prevalence in SSA.

Considering in particular STH and schistosomiasis, the authors pointed out the following that since the global prevalence of STH infections was first estimated by Stoll over 60 years ago, the overall prevalence of the STH infections is believed to have remained relatively constant in SSA, whereas it has diminished elsewhere in the developing world. Today, between one-quarter and one-third of SSA's population is affected by one or more STH infections, with children, especially school-aged children, disproportionately affected. Of the estimated 181 million school-aged children in SSA, almost one-half (89 million) are infected with hookworm, ascariasis, trichuriasis, or some combination of these STH infections. Hookworm infection ("hookworm"). Hookworm is the most common STH infection and the most common NTD in SSA. It is also one of the most important in terms of disease burden, accounting for up to one-third of the total burden from NTDs in SSA (see Table 3). Based on previous estimates derived in 2002. it is estimated that 198 million people in SSA are infected with hookworm (29\% of the region's population), including 40-50 million school-aged children. Approximately one-third of the world's hookworm today occurs in SSA, with the greatest number of cases occurring in Nigeria (38 million) and the Democratic Republic of Congo (DRC, 31 million), followed by Angola, Ethiopia, and Ivory Coast (10-11 million)). Hookworm is the most widely distributed NTD in SSA and it is pervasive throughout the region (including both rural and urban areas) except in some parts of extreme southern Africa. Two areas are particularly notable for their high hookworm prevalence and intensity compared to other helminth infections, namely coastal regions, and areas of extremely high temperatures (where land surface temperatures exceed $37-40^{\circ} \mathrm{C}$ ), including those near the Sahel such as Cameroon, Chad, and Mali. For instance, it is estimated that approximately $90 \%$ of the 50 million school-aged children with hookworm are at risk for coincident co-infection with falciparum malaria. of the world's 207 million estimated cases of schistosomiasis, 93\% occur in SSA (192 million), with the largest number in Nigeria (29 million) followed by the United Republic of Tanzania (19 million), DRC and Ghana (15 million each). Approximately 76\% of the population in SSA lives near rivers, lakes, and other water bodies contaminated with snail intermediate hosts. Those living near dam reservoirs are at particular risk, and SSA has several examples where the infection has emerged or where there has been a dramatic rise in the prevalence of schistosomiasis as a result of irrigation project construction. Climate change and global warming may also be factors. The highest prevalence and intensities of human schistosomiasis occur in schoolaged children, adolescents, and young adults who also suffer from the highest morbidity and mortality. There are two major forms of schistosomiasis found in SSA. Approximately two-thirds of the schistosomiasis cases are due to infection caused by Schistosoma haematobium, which represents an important cause of severe urinary tract disease. In 2000, van der Werf et al. estimated that 70 million and 32 million individuals out of 682 million people in SSA had experienced hematuria and dysuria, respectively, within the last two weeks. S. haematobium produces bladder wall pathology in approximately 18 million people in SSA, and 10 million people suffer from hydronephrosis. Renal failure accounts for a large percentage of the estimated 150,000 deaths from urinary tract schistosomiasis in SSA, and there is also a significant association between major bladder wall pathology and squamous cell carcinoma. A significant percentage of women and men with urinary schistosomiasis acquire genital ulcers and other lesions. In the former, urogenital schistosomiasis is a significant cause of poor reproductive health, including sexual dysfunction and infertility. Genital schistosomiasis also promotes the horizontal transmission of HIV/AIDS in SSA. Intestinal schistosomiasis from $S$. mansoni causes most of the remaining cases in SSA. An estimated 4.4 million people with $S$. mansoni have bloody diarrhea and bowel ulceration, and 8.5 million develop hepatomegaly and/or associated periportal liver fibrosis, portal hypertension, and hematemesis from 
S. mansoni infection, with approximately 130,000 deaths. S. intercalatum causes a second form of intestinal schistosomiasis, but with a restricted distribution in West and Central Africa. The current disease burden calculations for schistosomiasis range between 1.7 and 4.5 million DALYs lost annually (1.6 and 4.2 million DALYs in SSA), but these current estimates do not fully consider the general morbidities outlined above.

Table 3. Disease Burden (DALYs) in SSA Resulting from the NTDs.

\begin{tabular}{|c|c|c|c|}
\hline Disease & $\begin{array}{l}\text { Estimated Global } \\
\text { Disease Burden in } \\
\text { DALYs }\end{array}$ & $\begin{array}{l}\text { Estimated \% } \\
\text { Disease Burden in } \\
\text { SSA }\end{array}$ & $\begin{array}{l}\text { Estimated SSA Disease } \\
\text { Burden in DALYs }\end{array}$ \\
\hline Hookworm & 1.5-22.1 million & $34 \%$ & 0.5-7.5 million \\
\hline Schistosomiasis & 1.7-4.5 million & $93 \%$ & 1.6-4.2 million \\
\hline Ascariasis & 1.8-10.5 million & $21 \%$ & 0.4-2.2 million \\
\hline Lymphatic filariasis & 5.8 million & $35 \%$ & 2.0 million \\
\hline Trichuriasis & 1.8-6.4 million & $27 \%$ & 0.5-1.7 million \\
\hline $\begin{array}{l}\text { Human African } \\
\text { trypanosomiasis }\end{array}$ & 1.5 million & $100 \%$ & 1.5 million \\
\hline Trachoma & 2.3 million & $52 \%$ & 1.2 million \\
\hline Onchocerciasis & 0.5 million & $99 \%$ & 0.5 million \\
\hline Leishmaniasis & 2.1 million & $18 \%$ & 0.4 million \\
\hline Leprosy & 0.2 million & $14 \%$ & 0.02 million \\
\hline Dengue & 0.6 million & $<1 \%$ & 0.005 million \\
\hline Total NTDs & $\leq 56.6$ million & $15 \%-37 \%$ & 8.6 million-21.2 million \\
\hline
\end{tabular}

DALY estimates for STH infections and schistosomiasis were obtained by adjusting a wide range of available global estimates according to the percentage of the total number of cases that occur in SSA, while for the other NTDs the disease burdens were quoted directly from WHO estimates.

Apart from NTDs, other groups of emerging diseases are also of utmost importance in SSA. These diseases include for instance, Non Communicable Diseases (NCDs). A study was carried out in Mozambique in relation to NCDs, entitled: "Non-communicable diseases in Mozambique: risk factors, burden, response and outcomes to date." Mozambique was described in that study as one of the poorest countries in the world being ranked 184 out of 187 on the Human Development Index. Gross Domestic Product (GDP) per capita was International US\$ 1,000 and GDP (real growth) in 2010 was $7 \%$. In parallel to this economic development the rate of urbanization was estimated to be $4 \%$ with $31 \%$ of the total population living in urban areas in 2011. In comparison to other countries in Southern Africa Mozambique is currently one of the least urbanized countries. However, by 2025 it is estimated that it will be the fourth most urbanized. The four main NCDs, cardiovascular disease (CVD), cancer, diabetes and chronic obstructive pulmonary disease (COPD) share four common risk factors, tobacco use, the harmful use of alcohol, poor diet and lack of physical activity.

In "The emerging epidemic of obesity in developing countries", the authors stated that the pandemic is growing at such a pace that prevalence statistics become rapidly outdated. Detailed data on obesity from two nationwide surveys were available for Gambia, a small West African country on the southern edge of the Sahara that is typical of many nations in sub-Saharan Africa with respect to the epidemic. Gambia is in the early phases of the demographic and nutritional transitions described by Popkin. Like many such countries urbanization is increasing rapidly with a constant passage of

\footnotetext{
${ }^{4}$ Silva-Matos \& Beran, 2012

${ }^{5}$ Prentice, 2006
} 
young people from the rural areas to seek education and work in urban areas. In 1995, a representative survey was conducted on 6048 rural and urban adults (>15 years) from 73 communities using multistage stratified cluster sampling based on the 1993 national census. A second survey with similar results was conducted on 5373 adults in 1996-97. In the later survey the overall obesity rate was recorded as just $4 \%$ but this concealed major variations. In the rural population, obesity was essentially undetectable in men $(<0.2 \%$ prevalence) and only detectable in women $>35$ years at a prevalence of $<3 \%$. Among urban men the obesity rate was $<2 \%$ but amongst women it was much higher.

Still concerning non-communicable diseases, and in a study whose topic was "Noncommunicable diseases in sub-Saharan Africa: where do they feature in the health research agenda? ${ }^{6}$ ", it was mentioned that non-communicable diseases were not a high priority area for health research and development in the SSA countries. The estimates in the 1990 Global Burden of Disease study suggested that non-communicable diseases accounted for only $14 \%$ of the total burden in subSaharan Africa, and for just under a third in adults aged 15-59 years. Nonetheless, in absolute terms the estimates also suggested that the probability of death from non-communicable diseases was higher in sub-Saharan Africa than in Established Market Economies. Because of the lack of reliable mortality and morbidity data from SSA, these estimates were heavily based on assumptions and extrapolations. In at least three areas of the United Republic of Tanzania, one of Africa's poorest countries, the probabilities of death from non-communicable diseases were indeed higher than in Established Market Economies. Additionally, among adults, the age-specific death rates from non-communicable diseases were substantially higher in all age groups (i.e. 15-29 years, 30-44 years, and 45-59 years) in these three areas of the country than in the Established Market Economies. On the other hand, noncommunicable diseases account for $15-25 \%$ of all adult deaths (i.e. in persons aged 15-59 years) in the Tanzanian areas covered by the demographic surveillance system, a much smaller proportion than the $67 \%$ (men) to $80 \%$ (women) in the Established Market Economies.

Other emerging diseases or epidemics in SSA, such as the one related to Diabetic retinopathy (DR), were studied by other groups or researchers. This was the case with the study on "Diabetic retinopathy in sub-Saharan Africa: meeting the challenges of an emerging epidemic." A study in which the authors stated that diabetes causes visual impairment (VI) through early-onset cataract and diabetic retinopathy (DR), a progressive disease of the retinal microvasculature. Cataract and DR are the second and sixth leading causes of global VI, respectively. In a recent population-based survey ( $\mathrm{n}=4$ 4,414) in N akuru, $\mathrm{K}$ enya , identified a prevalence of 'any DR' of 35.9\% (95\% CI: 29.7, 42.6) and of 'severe non proliferative DR or PDR' of 13.9\% (95\% CI: 10.0, 18.8) in 277 people with diabetes [Bastawrous, personal communication]. Clinic-based studies report a wide range of prevalence, often with higher levels of sight-threatening disease, but these are subject to bias. The proportion of any visual impairment (VI) in African populations due to DR is largely unknown. A recent population-based study from Cape Town, South Africa, of visual loss using WHO methods, identified DR as the cause of $8 \%$ of blindness and $11 \%$ of severe visual loss in persons' $\geq 50$ years.

Concerning emergence of HIV as well as reemergence of tuberculosis, the "Tuberculosis case fatality rates in high HIV prevalence populations in sub-Saharan Africa" study was carried out in some SSA countries. The study was aiming at analysing the extent of the increased tuberculosis case fatality rate (CFR) in high HIV prevalence populations in sub-Saharan Africa, the reasons for this increase and the causes of death, in order to identify possible ways of tackling this problem. The CFR in HIV-positive and HIV-negative patients with sputum smear-positive pulmonary tuberculosis were respectively $6.1 \%$ and $0.4 \%$ in Cote d'Ivoire, $18 \%$ and $10 \%$ in Zomba, Malawi, $29 \%$ and $8 \%$ in Ntcheu, Malawi, 14\% and 0.5\% in Johannesburg, South Africa, and 13\% and 0\% in Zaire. The lowest tuberculosis CFR in HIV-positive patients was reported in Ivory Coast and should be interpreted

\footnotetext{
${ }^{6}$ Unwin et al, 2001

${ }^{7}$ Burgess, Msukwa, \& Beare, 2013

${ }^{8}$ Mukadi, Maher, \& Harries, s. d.
} 
cautiously given that one-third of the HIV-positive patients were lost to follow-up. A study in Malawi in patients with sputum smear- negative pulmonary tuberculosis reported a higher CFR in HIVpositive (59\%) than in HIV-negative (26\%) individuals. For comparison, the corresponding CFRs in sputum smear-positive patients were 18\% in HIV-positive and 10\% in HIV-negative patients.

Keeping talking about HIV, a review that was intended to provide some key examples of emerging HIV and AIDS pharmacotherapy issues, challenges, and priorities within resource-limited subSaharan African countries, and highlight how they contrast with those of the developed world, was carried out. The topic of the study was: "HIV Pharmacotherapy Issues, Challenges, and Priorities in sub-Saharan African Countries." In terms of challenges to resource provision for supporting HIV and AIDS pharmacotherapy, it was revealed concerning drug financing that the Gross National Products (GNPs) per capita in most sub-Saharan African countries was low (see Table 4). Therefore, until recently, antiretroviral drugs have been beyond the economic reach of most. Owing to economic hindrances, most of these countries have used a variety of strategies to address the issue of equitable access to antiretrovirals. Such strategies include adopting the World Trade Organization's Trade Related Intellectual Properties (WTO- TRIP) safeguards into national legislation, actively encouraging generic competition, seeking differential pricing of drugs from international suppliers, creating high volume and demand through regional procurement, and encouraging local production through licensing and technology transfer. Approximately 90\% of newly infected children in 2006 lived in SSA countries, where the major route of infection is through mother-to-child transmission. As a result of the limited accessibility to antiretroviral therapy, the emphasis in SSA countries is still largely on the prevention of HIV transmission. The HIV and AIDS epidemic has highlighted the weakness of health care systems in resource-limited SSA countries in terms of referral systems, human resources, and laboratory capacity, as well as drug procurement and supply chains. Lack of capacity in the educational system has led to an overall shortage of qualified health personnel including pharmacists and pharmacy staff.

T able 4. Pharmacy Infrastructure in 5 African Countries in 1999

\begin{tabular}{|c|c|c|c|c|c|}
\hline & Botswana & Malawi & Swaziland & Zambia & Zimbabw \\
\hline Total population (million) & 1.5 & 11.0 & 0.9 & 10.2 & 12.4 \\
\hline GNP per capita (\$US) & 6872 & 586 & 3987 & 756 & 2876 \\
\hline $\begin{array}{l}\text { Number of pharmaceutical } \\
\text { manufacturers/wholesalers }\end{array}$ & 3 & 3 & 0 & 5 & 22 \\
\hline Number of registered drug products & 340 & 1500 & No DRA & 1200 & 1700 \\
\hline Number of registered pharmacists & 160 & 60 & 33 & 100 & 530 \\
\hline $\begin{array}{l}\text { Number of registered pharmacy } \\
\text { outlets }\end{array}$ & 63 & 16 & 13 & 78 & 240 \\
\hline
\end{tabular}

Adapted from World Health Organization study of drug regulatory authorities, 1999. GNP indicates gross national product; DRA, drug regulatory authority

From July to January 2013, MHRL conducted first-tier lateral flow immunoassay (LFI) tests of blood samples from all 932 outpatiente 5 years of age who had been clinically examined at the hospital, were found to have febrile illness, and consented to having blood drawn for laboratory

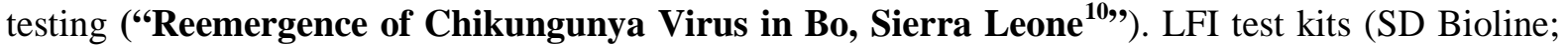

\footnotetext{
${ }^{9}$ (Maponga, Ma, Slish, \& Morse, 2007)

${ }^{10}$ Ansumana et al., 2013
} 
Standard Diagnostics, Inc., Seoul, South Korea) were used for diagnosis of IgM against CHIKV; IgG and IgM against dengue virus and hepatitis A virus; hepatitis B virus surface antigen, hepatitis C virus, HIV-1/2, and antibodies against these viruses; and IgG and IgM against Leptospira spp., Salmonella enterica Serovar typhi, and syphilis. Most patients reported that they had sought medical care within several days after the onset of their febrile illnesses. Levels of IgM against CHIKV are usually detectable by immunochromatographic methods within a few days after infection and persist for $\approx 3-4$ months. The LFI test kits for CHIKV were reported by the manufacturer to have a sensitivity of $97.1 \%$ and a specificity of $91.1 \%$ compared with those of ELISA. An independent evaluation found a sensitivity of $50.8 \%$ and a specificity of $89.2 \%$ for the kits; sensitivity ranged from $40.9 \%, 1-5$ days after onset of illness to $65.4 \%, 16-20$ days after onset. Specificity decreases after the first week. More than half of the cases tested during the first week of the surveillance program were positive by LFI for CHIKV. Thus, we notified the Sierra Leone Ministry of Health and Sanitation of a possible CHIKV outbreak. By January 10, 2013, 400 (42.9\%) of 932 febrile patients were positive by LFI for CHIKV. Ages of the 400 CHIKV IgM-positive patients ranged from 6 years to 85 years; 172 (43.0\%) were male patients. Of these 400 patients, $220(55.0 \%)$ reported arthralgia, 189 (47.3\%) chills, and 156 (39.0\%) headaches. Co-infections were common; 92 (23.0\%.) were co-infected with malaria, 37 (9.3\%) with HIV, 33 (8.3\%) with hepatitis B virus, and smaller numbers with hepatitis A, hepatitis C, tuberculosis, typhoid, and syphilis. Four CHIKV-positive samples were also positive for dengue.

A study to better understand the implications of the presence of body lice in some individuals, was carried in six countries ("Body lice as tools for diagnosis and surveillance of reemerging

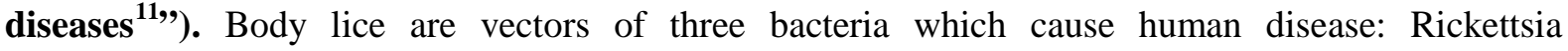
prowazekii, the agent of epidemic typhus; Bartonella quintana, the agent of trench fever; and Borrelia recurrentis, the agent of relapsing fever. A recrudescence of body lice is being observed as the numbers of individuals living under social conditions which predispose individuals to infestation have increased. Because this phenomenon may lead to the reemergence of infections transmitted by body lice, we aimed to assess the occurrence and prevalence of the three agents described above in more than 600 body lice collected from infested individuals in the African countries of Congo, Zimbabwe, and Burundi, in France, in Russia, and in Peru. The presence of the three bacteria in each louse was determined by specific PCR amplification, and the identities of the organisms detected were confirmed by determination of the nucleotide base sequences of the amplification products. Using this approach, we were able to confirm the presence of R. prowazekii in lice collected from refugees in Burundi, among whom typhus was epidemic, and the presence of B. quintana in lice collected from all locations except the Congo. B. recurrentis was never found. Molecular approaches are convenient tools for the detection and identification of bacterial DNA in body lice and for the epidemiological study of louse-borne bacteria from countries where no medical and biological laboratory facilities are available. The sensitivities of the PCR assays with the primers CS-877 and CS-1273, CSBAR-218 and CSBAR-698, and Bf1 and Br1 were estimated to be from 1 to 10 copies of the gene (DNA). For the detection of $B$. quintana, PCR amplification was performed with the primers CSBAR-218 and CSBAR-698, and the positivity was confirmed with the primers QHVE1 and QHVE3. For the detection of $R$. prowazekii, PCR amplification was performed with the primers CS-877 and CS-1273, and the positivity was confirmed with the primers 120-M59 and 120-797.

In addition to the high propensity of these infections, the populations in SSA as we have already said, also suffer significant problems of poverty and Inequality of access to care. This was demonstrated in the study entitled: "Poverty, inequality and health: the challenge of the double burden of disease in a non-profit hospital in rural Ethiopia. ${ }^{12}$ ” This study was aimed at describing disease patterns in a rural zone of Oromiya region, Ethiopia through a retrospective analysis of discharge records for 22,377 inpatients of St. Luke Hospital, Wolisso, Ethiopia in the 2005-2007 period. The leading cause of admission was childbirth, followed by injuries, malaria and pneumonia.

\footnotetext{
${ }^{11}$ Roux \& Raoult, 1999

${ }^{12}$ Accorsi et al., 2009
} 
Injuries were the leading cause of in-hospital deaths, followed by pneumonia, malaria, cardiovascular disease and AIDS. Vulnerable groups (infants, children and women) accounted for $73.3 \%$ of admissions. Most of the disease burden resulted from infectious diseases, the occurrence of which could be dramatically reduced by cost-effective preventive and curative interventions. Furthermore, a double burden of disease is already emerging at the early stage of the epidemiological transition, with a mix of persistent, emerging and re-emerging infectious diseases and increasing prevalence of chronic conditions and injuries. This will lead to fundamental changes in the volume and composition of demand for healthcare, with a more complex case mix and costlier service utilization patterns. The challenge is to address the double burden of disease, while focusing on poverty-related conditions and targeting vulnerable groups. Monitoring disease and service utilization patterns through routine hospital information systems can provide sustainable, low-cost support for evidence-based health practice.

The data and information provided by all the above mentioned studies, demonstrate widely that there exists a high degree of needs, but also demands, in regards to emerging and reemerging diseases in SSA, even if tremendous efforts have been made. We can indeed observe on the basis of epidemiological data and the above information that, the pressure due to these so-called emerging diseases both on populations and communities, as well as on the public health systems, is huge. Pressure which of course led to the different health systems, researchers and other stakeholders to become more involved in the search for solutions.

However, the search for appropriate solutions should nevertheless be accompanied by respect for universal and well recognized ethical standards. It is in that line that a study on ethical issues related to compulsory and voluntary screening was carried out in Kenya. The study whose subject was "Ethical issues evolving from patients' perspectives on compulsory screening for syphilis and voluntary screening for cervical cancer in Kenya ${ }^{13}$, revealed that real ethical challenges exist in the voluntary and compulsory screening approaches. Also, participants were more concerned about the benefits of the procedure and whether their dignity is respected than the compulsoriness of screening per se. The implication is for the policy makers to clarify in the guidelines how to manage ethical challenges, while at the operational level, providers need to be judicious to minimize potential harm to participants and families when screening for disease in women.

Still in Kenya, a study documented opportunities and challenges encountered in managing cervical cancer from the health care workers' perspectives. Kenya, like other developing countries is low in resource setting and is facing a number of challenges in the management of cervical cancer. A qualitative study (“An exploration of opportunities and challenges facing cervical cancer managers in Kenya ${ }^{14 \text { ") }}$ was conducted among cervical cancer managers who were defined as nurses and doctors involved in operational level management of cervical cancer. Four themes were identified. Patient related challenges included a large number of patients, in the late stage of the disease, low levels of knowledge on cancer of the cervix, low levels of screening and a poor attitude towards screening procedures. Individual health care providers identified a lack of specialized training, difficulty in disclosure of diagnosis to patients, a poor attitude towards cervical cancer screening procedures and a poor attitude towards cervical cancer patients. Health facilities were lacking in infrastructure and medical supplies. Some managers felt ill-equipped in technological skills while the majority lacked access to the internet. Mobile phones were identified as having great potential for improving the management of cervical cancer in Kenya.

In the meanwhile, a group of researchers in Ghana worked on Buruli ulcer, which is considered a re-emerging disease in West Africa where it has suffered neglect over the years, though children below the age of 16 years are the worst affected in most endemic regions. "Enhancing Buruli ulcer control in Ghana through social interventions: a case study from the Obom sub-district ${ }^{15}$ ” was

\footnotetext{
${ }^{13}$ Omondi Aduda \& Mkhize, 2014

${ }^{14}$ Kivuti-Bitok, Pokhariyal, Abdul, \& McDonnell, 2013

${ }^{15}$ Ahorlu, Koka, Yeboah-Manu, Lamptey, \& Ampadu, 2013
} 
the title of their study. According to them and due to delayed health seeking, the disease leads to disabilities resulting from amputation and loss of vital organs like the eye, leading to school dropout and other social and economic consequences for the affected family. The mode of transmission of Buruli ulcer is not known and there is no effective preventive vaccine for Buruli ulcer. Thus the only effective control tool is early case detection and treatment to reduce morbidity and associated disabilities that occur as a result of delayed treatment. It is therefore essential to implement interventions that remove impediments that limit early case detection; access to early effective treatment and this paper reports on such effort where the feasibility of social interventions to enhance Buruli ulcer control was assessed. At full implementation, treatment default and dropout reduced significantly from $58.8 \%$ and $52.9 \%$ at baseline to $1.5 \%$ and $1.5 \%$ respectively. The number of early case detection went up significantly.

In the same perspective but considering now the field of medical laboratory analyzes, considerable resources have been invested in recent years to improve laboratory systems in resource-limited settings. A study in which published reports were reviewed, major donor organizations were interviewed, and case studies of laboratory systems were conducted in 3 countries to assess how countries and donors have worked together to improve laboratory services was carried out. While infrastructure and the provision of services have seen improvement, important opportunities remain for further advancement. Implementation of national laboratory plans is inconsistent, human resources are limited, and quality laboratory services rarely extend to lower tier laboratories (eg, health clinics, district hospitals). Coordination within, between, and among governments and donor organizations is also frequently problematic. Laboratory standardization and quality control are improving but remain challenging, making accreditation a difficult goal. Host country governments and their external funding partners should coordinate their efforts effectively around a host country's own national laboratory plan to advance sustainable capacity development throughout a country's laboratory system. (Strengthening Laboratory Systems in Resource-Limited Settings ${ }^{16}$ )

Apart from the human resources issues, infrastructure or technology, Sub-Saharan Africa also faces enormous difficulties in terms of health-related information. This applies both with regard to the information towards the population and to the information directed to the service providers. And this is what was highlighted in a study that was carried out in South Africa and Uganda: "Meeting information needs of patients with incurable progressive disease and their families in South Africa and Uganda: multicentre qualitative stud ${ }^{17}$ " Five themes emerged from the data. (1) Information sources: a lack of information from general healthcare providers meant that patients and caregivers had to draw on alternative sources of information. (2) Information needs: patients and caregivers reported needing more information in the key areas of the causes and progression of the disease, its symptoms and treatment, and financial/social support. (3) Impact of unmet needs: poor provision of information had a detrimental effect on patients' and caregivers' ability to cope. (4) Communication: negative experiences of communication with general healthcare staff were reported (misinformation, secrecy, insensitivity). (5) Barriers to effective provision of information: barriers related to symptoms, culture, time constraints in hospital, and paternalism in general health care.

Yet all is not negative, not everything is reduced to failures and malfunctions. Many advances have been made all the same, even if people do not talk enough about them. In fact, a number of researches have been carried out, to enhance and reinforce practices in these domains. It is in this perspective that a group of researchers worked on the theme called "Health sector demand-side financial incentives in low- and middle-income countries: a systematic review on demand- and supply-side effects. ${ }^{18}$ " According to the authors, Demand-side financial incentive (DSF) is an emerging strategy to improve health seeking behavior and health status in many low- and middleincome countries. This narrative synthesis assessed the demand- and supply-side effects of DSF.

\footnotetext{
${ }^{16}$ Olmsted et al., 2010

${ }^{17}$ Selman et al., 2009

${ }^{18}$ Gopalan, Mutasa, Friedman, \& Das, 2014
} 
Forty-one electronic data bases were searched to screen relevant experimental and quasi-experimental study designs. Out of the 64 selected papers, 28 were eligible for this review and they described 19 DSF initiatives across Asia, Africa and Latin America. There were three categories of initiatives, namely long-run multi-sectoral programs or LMPs (governmental); long-run health-exclusive programs (governmental); and short-run health-exclusive initiatives (both governmental and nongovernmental). Irrespective of the nature of incentives and initiatives, all DSF programs could achieve their expected behavioral outcomes on healthcare seeking and utilization substantially. However, there existed a few negative and perverse outcomes on health seeking behavior and DSF's impact on continuous health seeking choices (e.g. bed net use and routine adult health check-ups) was mixed. Their effects on maternal health status, diarrhea, malaria and out-of-pocket expenditure were underexplored; while chronic non-communicable diseases were not directly covered by any DSF programs. DSF could reduce HIV prevalence and child deaths, and enhance nutritional and growth status of children. The direction and magnitude of their effects on health status was elastic to the evaluation design employed. On health system benefits, despite prioritizing on vulnerable groups, DSF's substantial effect on the poorest of the poor was mixed compared to that on the relatively richer groups. Though DSF initiatives intended to improve service delivery status, many could not optimally do so, especially to meet the additionally generated demand for care. Causal pathways of DSF's effects should be explored in-depth for mid-course corrections and cross-country learning on their design, implementation and evaluation. More policy-specific analyses on LMPs are needed to assess how 'multisectoral' approaches can be cost-effective and sustainable in the long run compared to 'health exclusive' incentives.

Training/capacity building of human resources thus appears as one of the main challenges. It is true that the one of infrastructure and technology is equally garish. Reason why Nigeria for instance, has opted for the training of service providers and more particularly of field epidemiologists. The program was presented in a paper ("Training and Service in Public Health, Nigeria Field Epidemiology and Laboratory Training, 2008 - 2014 ${ }^{19}$ ”) in which strategies and results were described. According to the authors, the health workforce is one of the key building blocks for strengthening health systems. There is an alarming shortage of curative and preventive health care workers in developing countries many of which are in Africa. Africa resultantly records appalling health indices as a consequence of endemic and emerging health issues that are exacerbated by a lack of a public health workforce. In low-income countries, efforts to build public health surveillance and response systems have stalled, due in part, to the lack of epidemiologists and well-trained laboratorians. The Nigeria Field Epidemiology and Laboratory Training Program (NFELTP) was established in October 2008 as an in-service training program in field epidemiology, veterinary epidemiology and public health laboratory epidemiology and management. The first cohort of NFELTP residents began their training on October 20, 2008 and completed their training in December, 2010. The program was scaled up in 2011 and admitted 39 residents in its third cohort. The program has admitted residents in six annual cohorts since its inception, admitting a total of 207 residents as of 2014 covering all the States. In addition, the program has trained 595 health care workers in short courses. Since its inception, the program has responded to 133 suspected outbreaks ranging from environmental related outbreaks, vaccine preventable diseases, water and food borne diseases, zoonoses, (including suspected viral hemorrhagic fevers) as well as neglected tropical diseases. The program has been involved in polio eradication efforts through its National Stop Transmission of Polio (NSTOP). The commencement of NFELTP was a novel approach to building sustainable epidemiological capacity to strengthen public health systems especially surveillance and response systems in Nigeria.

Countries in SSA regions, although late in the matter, have nevertheless taken some positive steps in terms of new medical technologies. Thus to illustrate this, and in a study conducted in Cameroon entitled "Local innovation for improving primary care cardiology in resource-limited African

\footnotetext{
${ }^{19}$ Nguku et al, 2014
} 
settings: an insight on the Cardio Pad project in Cameroon ${ }^{20}$,, it was said that to overcome the current health workforce shortage in African countries, some strategies such as the task shifting and telehealth have been suggested as means for rapidly increasing access to prevention and curative services for emerging cardiovascular diseases (CVD) and NCDs. In addition, cardiovascular disease (CVD) is an emerging threat to the health of populations in Africa. With the inadequate health infrastructures, understaffed and underfunded health systems, African countries are ill-prepared to cope with the increasing demand of care for CVD, particularly for populations in remote and underserved rural areas, where $60 \%$ of the population currently reside. Task shifting and telehealth have been suggested as strategies to overcome the current health workforce shortage in African countries, and to increase access to prevention and curative services for emerging CVD. However, strategies for promoting their incorporation into the existing health systems, have yet to be developed. The Cardio Pad initiative (originating from Cameroon) seeks to provide appropriate solutions to improve the application of telemedicine for CVD prevention and control in remote African settings. The Cardio Pad is a telecardiology device which provides a number of advantages in terms of cost, ease of use, autonomy and reduced technology requirements. It is a fully touch screen medical device which enables cardiac tests such as electrocardiograms (ECG) to be performed in remote underserved areas (rural areas for instance), while the test results are transferred wirelessly via mobile phone connection, to specialist physicians who can interpret them and provide assistance with case management.

Discussion: Without any willingness of stigmatization, two countries appear on the basis of most data described above, to have the largest number of emerging diseases, in particular as part of NTD cases and possibly disease burden. Nigeria is estimated to have the highest prevalence of helminth infections such as hookworm, schistosomiasis, ascariasis, trichuriasis, and LF in SSA, as well as the second highest registered prevalence of leprosy. In addition, arboviral and other zoonotic infections are common in Nigeria, including yellow fever, rabies, and toxoplasmosis, while Buruli ulcer is found in the southern and southeastern areas of the country. Similarly, DRC exhibits the highest prevalence of HAT and leprosy in SSA, as well as the second highest prevalence of hookworm infection and trichuriasis (and possibly LF), and the third highest prevalence of schistosomiasis and ascariasis. Together, Nigeria and DRC account for approximately one-third of the helminth infections and leprosy in SSA, as well as up to one-fifth of the cases of HAT. It should otherwise be pointed out that these countries are the most populated and the one with the highest size respectively, in SSA. A targeted approach for these two countries could make a substantial impact on Africa's overall NTD disease burden. In "Defining an emerging disease" ${ }^{21}$ " the authors underlined that the definition of emerging disease is not straightforward, as there are several different types of disease emergence. For example, there can be a 'real' emergence of a brand new disease, such as the emergence of bovine spongiform encephalopathy in the 1980s, or a geographic emergence in an area not previously affected, such as the emergence of bluetongue in northern Europe in 2006. In addition, disease can emerge in species formerly not considered affected, e.g. the emergence of bovine tuberculosis in wildlife species since 2000 in France. There can also be an unexpected increase of disease incidence in a known area and a known species, or there may simply be an increase in our knowledge or awareness of a particular disease. What all these emerging diseases have in common is that human activity frequently has a role to play in their emergence... They further continued by saying that globalisation and population growth will continue to affect the epidemiology of diseases in years to come and ecosystems will continue to evolve.

In most of the papers reviewed, main issues raised were on one hand related to the pressure put on the facility by patients and caretakers of these patients, and on the other hand, the global matter of information.

\footnotetext{
${ }^{20}$ Noubiap, Jingi, \& Kengne, 2014

${ }^{21}$ Moutou \& Pastoret, 2015
} 
Concerning pressure put on health facilities and the health system, it was for instance demonstrated during the study on Buruli Ulcer ("Enhancing Buruli ulcer control in Ghana through social interventions: a case study from the Obom sub-district ${ }^{22}$ ), that it was not only possible to increase Buruli Ulcer early case detection and diagnosis but also to effectively treat patients on outpatient basis at health centers to significantly reduce in-patient pressure on the health facilities in terms of space for admission. Hospital admission do not only put pressure on the health facility but also on the family caretakers who have to stay on 'admission' with the patients for as long as it takes to get the patient discharged from the hospital. In this study, caretakers were not even accompanying patients to the health center and this made it possible for them to go about their normal daily life while patients received effective treatment from the health center. In the study related to "Meeting information needs of patients with incurable progressive disease and their families in South Africa and Uganda: multicentre qualitative study ${ }^{23}$,, it was the first time in Africa that a study tried to understand experiences of communication and information sharing among patients with incurable progressive disease and their informal caregivers and to identify their unmet needs. Most patients and caregivers lacked information and had unanswered questions about their conditions. This situation seemed to exist in spite of them drawing on a wide range of sources for information. Patients and caregivers often considered general medical services to be inadequate at providing accessible information that met their needs... Poor knowledge and provision of information adversely affected patients' and caregivers' ability to cope with their situation, directly affecting caregivers' ability to care for patients, as well as patients' ability to care for themselves and plan for the future. For both groups, not having the information they needed was related to anxiety about the disease and the future.

Despite these many failures, progress has been made all the same, not only in the field of financing, with demand-side financial incentives ("Health sector demand-side financial incentives in lowand middle-income countries: a systematic review on demand- and supply-side effects ${ }^{24}$.") but also in the field of training of epidemiologists, to reinforce public surveillance ("Training and Service in Public Health, Nigeria Field Epidemiology and Laboratory Training, $2008-2014^{25}$ ”). In a final note, Technological innovations in medical domains, are nevertheless to be credited to researchers and scientists from the SSA region. We would particularly mention the case of the Cardio Pad, which was developed by a young Cameroonian, to rapidly increase access to prevention and curative services for emerging cardiovascular diseases (CVD) and NCDs.

Conclusion: Emerging and reemerging infections or diseases, really represent a major public health issue in SSA, but also a major economic problem, through the disease burden that they represent. This review has enabled us to get an idea of the magnitude of the problem, epidemiologically and as regard to the disease burden in terms of Disability Adjusted Life Year (DALY). In fact, populations in the SSA region are constantly plagued by threats and epidemics such as malaria, HIV, Ebola, chikungunya, the schistosomiasis, helminths and other non-communicable diseases. These different diseases and infections that endanger the lives of these populations are most often the result of human action in the field of agriculture, dam construction, ecological changes, and so one and so forth. As such it can be also by human action that these scourges can be eradicated. Challenges exist in terms of the resurgence of these diseases, as well as their magnitude, as regards to epidemiological data, burden, and capacity of health systems to meet the needs and demands of the populations, but also ability of each other to adapt to these new situations.

Fortunately, several initiatives are underway or have been taken to resolve some of these situations. This was particularly the case in terms of building human resource capacity, better financing of demand, but also in terms of technological innovation.

\footnotetext{
${ }^{22}$ Ahorlu et al. 2013

${ }^{23}$ Selman et al, 2009

${ }^{24}$ Gopalan et al, 2014

${ }^{25}$ Nguku et al, 2014
} 


\section{References}

[1]. Accorsi, S., Kedir, N., Farese, P., Dhaba, S., Racalbuto, V., Seifu, A., \& Manenti, F. (2009a). Poverty, inequality and health: the challenge of the double burden of disease in a non-profit hospital in rural Ethiopia. Transactions of the Royal Society of Tropical Medicine and Hygiene, 103(5), 461-468. https://doi.org/10.1016/j.trstmh.2008.11.027

[2]. Acheson, R. M. (1978). The definition and identification of need for health care. Journal of Epidemiology and Community Health, 32(1), 10-15.

[3]. Ahorlu, C. K., Koka, E., Yeboah-Manu, D., Lamptey, I., \& Ampadu, E. (2013). Enhancing Buruli ulcer control in Ghana through social interventions: a case study from the Obom sub-district. BMC Public Health, 13, 59. https://doi.org/10.1186/1471-2458-13-59

[4]. Ansumana, R., Jacobsen, K. H., Leski, T. A., Covington, A. L., Bangura, U., Hodges, M. H., ... Stenger, D. A. (2013). Reemergence of chikungunya virus in Bo, Sierra Leone. Emerging Infectious Diseases, 19(7), 1108-1110. https://doi.org/10.3201/eid1907.121563

[5]. Burgess, P. I., Msukwa, G., \& Beare, N. A. V. (2013). Diabetic retinopathy in sub-Saharan Africa: meeting the challenges of an emerging epidemic. BMC Medicine, 11, 157. https://doi.org/10.1186/1741-7015-11-157

[6]. Gopalan, S. S., Mutasa, R., Friedman, J., \& Das, A. (2014). Health sector demand-side financial incentives in low- and middle-income countries: a systematic review on demand- and supply-side effects. Social Science \& Medicine (1982), 100, 72-83. https://doi.org/10.1016/j.socscimed.2013.10.030

[7]. Hotez, P. J., \& Kamath, A. (2009). Neglected Tropical Diseases in Sub-Saharan Africa: Review of Their Prevalence, Distribution, and Disease Burden. PLOS Negl Trop Dis, 3(8), e412. https://doi.org/10.1371/journal.pntd.0000412

[8]. Kivuti-Bitok, L. W., Pokhariyal, G. P., Abdul, R., \& McDonnell, G. (2013). An exploration of opportunities and challenges facing cervical cancer managers in Kenya. BMC Research Notes, 6, 136. https://doi.org/10.1186/1756-0500-6-136

[9]. Maponga, C. C., Ma, Q., Slish, J. C., \& Morse, G. D. (2007). HIV pharmacotherapy issues, challenges, and priorities in sub-Saharan African countries. Topics in HIV Medicine: A Publication of the International AIDS Society, USA, 15(3), 104-110.

[10]. Morse, S. S. (1995). Factors in the emergence of infectious diseases. Emerging Infectious Diseases, 1(1), 7-15.

[11]. Moutou, F., \& Pastoret, P.-P. (2015). Defining an emerging disease. Revue Scientifique Et Technique (International Office of Epizootics), 34(1), 41-52.

[12]. Mukadi, Y. D., Maher, D., \& Harries, A. (s. d.). Tuberculosis case fatality rates in high HIV prevalence popu... $\square$ : AIDS. Consulté 25 septembre 2016, à l'adresse http://journals.lww.com/aidsonline/Fulltext/2001/01260/Tuberculosis_case_fatality_rates_in_high_HIV.2.aspx [13]. Nguku, P., Oyemakinde, A., Sabitu, K., Olayinka, A., Ajayi, I., Fawole, O., ... Nasidi, A. (2014). Training and service in public health, Nigeria Field Epidemiology and Laboratory Training, 2008 - 2014. The Pan African Medical Journal, 18 Suppl 1, 2. https://doi.org/10.11694/pamj.supp.2014.18.1.4930

[14]. Noubiap, J. J. N., Jingi, A. M., \& Kengne, A. P. (2014). Local innovation for improving primary care cardiology in resource-limited African settings: an insight on the Cardio Pad(®) project in Cameroon. Cardiovascular Diagnosis and Therapy, 4(5), 397-400. https://doi.org/10.3978/j.issn.2223-3652.2014.10.01

[15]. Olmsted, S. S., Moore, M., Meili, R. C., Duber, H. C., Wasserman, J., Sama, P., ... Hilborne, L. H. (2010). Strengthening laboratory systems in resource-limited settings. American Journal of Clinical Pathology, 134(3), 374-380. https://doi.org/10.1309/AJCPDQOSB7QR5GLR

[16]. Omondi Aduda, D. S., \& Mkhize, N. (2014). Ethical issues evolving from patients' perspectives on compulsory screening for syphilis and voluntary screening for cervical cancer in Kenya. BMC Medical Ethics, 15, 27. https://doi.org/10.1186/1472-6939-15-27

[17]. Prentice, A. M. (2006). The emerging epidemic of obesity in developing countries. International Journal of Epidemiology, 35(1), 93-99. https://doi.org/10.1093/ije/dyi272

[18]. Roux, V., \& Raoult, D. (1999). Body lice as tools for diagnosis and surveillance of reemerging diseases. Journal of Clinical Microbiology, 37(3), 596-599. 
Texila International Journal of Public Health

Volume 4, Issue 4, Dec 2016

[19]. Selman, L., Higginson, I. J., Agupio, G., Dinat, N., Downing, J., Gwyther, L., ... Harding, R. (2009). Meeting information needs of patients with incurable progressive disease and their families in South Africa and Uganda: multicentre qualitative study. BMJ (Clinical Research Ed.), 338, b1326.

[20]. Silva-Matos, C., \& Beran, D. (2012). Non-communicable diseases in Mozambique: risk factors, burden, response and outcomes to date. Globalization and Health, 8, 37. https://doi.org/10.1186/1744-8603-8-37

[21]. Unwin, N., Setel, P., Rashid, S., Mugusi, F., Mbanya, J.-C., Kitange, H., ... Alberti, K. G. M. M. (2001). Noncommunicable diseases in sub-Saharan Africa: where do they feature in the health research agenda? Bulletin of the World Health Organization, 79(10), 947-953. https://doi.org/10.1590/S0042-96862001001000008 